that if medical men would only send in such offers as would enable them to discharge their duties with honour to themselves, and justice to the poor, the evils of the tender system would be remedied without the assistance of the Legislature.

Sir Jory Frfe, who moved that the annual subscription be reduced from one pound to ten shillings, said, that the resolution which he had the honour to propose clearly demonstrated the increasing prosperity of the North of England Medical Association. The Association now numbered a hundred and sixty members - the funds were good-their power was growing; and it was clear, that a body so influential, organized, unanimous, and powerful, must soon make an impression on public opinion, and, consequently, upon the Legislature. (Applause.) After having replied to Mr. Morrison's remarks on the tender system, Sir John Fife concluded by observing, that when he reflected on the past proceedings and future prospects of the Association, and that their ruling principle was to raise the qualification, elevate the social position, and increase the usefulness of the medical profession, he could not harbour a doubt of their ultimate and complete success. (Applause.)

The resolution was carried without opposition.

Dr. K worr, of Newcastle, with a view of rendering the union of the Association more efficient in the cause of Medical Reform, moved sereral instructions to the Council ; one relative to the establishment of local associations in the North of England; another inviting members of the profession to communicate their grievances, or plans of reform; a third to define the qualification of candidates for enrolment in the Association, and of practitioners for consultation with any of its members. His fourth instruction was, that all resnlutions be printed and circulated among the members, previous to meetings being held; and that a time be fixed, after which no resolution shall be received for the ensuing meeting.

Dr. Ell10t, of Gateshead, seconded the motion of Dr. Knott, and suggested that the Council should frame a code of law for the general guidance of the profession.

Mr. Morrisos then made some observations on the working of the Anatomy Act, which, he said, was rendered almost inoperative from the circumstance that the act left it uncertain in whom the legal possession of unclaimed bodies rested.

Dr. Ellror read an interesting paper "On Scientific Medicine : its Relations and Claims on Society at large ;" and the meeting separated, after the presentation of thanks to their venerated President, Dr. Headlam.

\section{Case of strangulated femoral hernia.} BY MR. BANNER,

Burgeon to the Liverpool North Hospital.

Margaret Maxin, wet. 40, was seized with symptoms of strangulated femoral hernia in February last, when Mr. Banner was called to see the case, in consultation with $\mathrm{Mr}$. Bradshaw. The history given was as follows :-She had a rupture in the left groin, after the delivery of her first child, which was in 1823; she had been obliged to wear a truss almost constantly ever since; occasionally, when sho had been without her truss, or when she was using much exertion, the rupture came down, and she had slight difficulty in returning it. On the 10th of February, after having washed from early in the morning until evening, the rupture came down, and she was unable to return it; early in the moming of the 11th she felt sick and faint; there was pain in the tumout, and slight griping pain in the bowels. These symptoms increased in severity until the night of the 11th, when Mr. Bradshaw was sent for. He found the patient with a small hard hernia in the left groin; which was irreducible; the pulse was weak and very quitk, and there was frequent vomiting. After endeavourinis in vain; to persuade her to go into the hospital, he had recouks to the usual means for the reduction of the hernia ; his efforts, however, were fruitless. It was not until the afternoon of the 12 th inst. that she consented to the operation, which was performed by Mr. Banner in the usual manner. The cellular membrane covering the hernia was found very indurated and thick; within the sac were about three drachms of a thick fluid, which much resembled cream in colour and consistency-it was free from smell, and on its surface there floated oily globules; there was a small hard flat piece of omentum within the sac, and also a small knuckle of intestine, which was dark coloured, but briglit, and such as was thought would maintain its vitality. It was, with the omentum (which slightly adhered) returned into the abdominal cavity. A mixture, composed of sulphate of magnesia and infusion of roses, was given every two hours. Soon after the operation the romiting and pain ceased, and before morning she had parted with three evacuations by the rectum; the bowels continued to act regularly during the first four days after the operation. The wound looked slightly inflamed; the discharge from it was healthy; on the night of the fourth day a sudden gush of fluid came through the wound, with a great noise, which was described like that of wind passing through an opening; the fluid discharged was very offensive, and yellow in colour.

On the fifth day after the operation, Mr. Banner saw the case again, when it was ascertained that there was an escape of fæces and flatus; the wound looked sloughy, and the patient appeared to be suffering from a good deal of constitutional irritation; stool passed through the rectum at the same time that it passed through the wound. In a week after this event, a large slough was thrown off, which appeared to consist principally of the sac and cellular membrane; the wound soon assumed a healthy appearance; the discharge of foeculent matter rapidly diminished, and in a few days entirely ceased; the stools passed freely through the rectum, and in three veeks the wound was quite healed. The woman was nearly five months advanced in pregnancy; she was delivered, at her proper time, of a living child, which made her fifteenth.

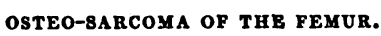

Catherine Williams, at. 17, was admitted into the Liverpool North Hospital on the 3 lst of July, 1840, under Mr. Banner's care, with osteo-sarcoma of the lower end. of the left femur. She stated that seven months previous to her admission she felt pain and stiffness in the left knee; the joint began to swell, and very soon attained a great size; the pain increased in proportion to the growth of the swelling, and at last she was obliged to leave her situation as a servant. She had always, previous to this attack, good health. She had applied to the dispensary for relief, and had leeches, cupping glasses, and blisters applied; the disease increased, and she came into the hospital. At this time there was considerable emaciation; the countenance pallid and anxious, the pulse quick and feeble, the secretions scanty; the knee was enormously swelled, and very hard; the swelling existed more to the inside of the knee than the outside; the foot was turned out, and the limb had an appearance of lateral dislocation; there was a feeling of fluctuation, deep and indistinct; the patella appeared pressed to the outside of the knee; there was considerable motion in the joint, and the patella could be readily moved. The pain, which at the time of her admission was inconsiderable, was referred to the ham; this pain increased at night, and during sleep she was often awoke by a starting or shooting of the limb; the swelling was free from discolouration, and the veins were not visible.

This case presented much the appearance of chronic inflammation of the knee joint; but the want of a distinct feeling of fuctuation, and the extraordinary hardness, rendered the diagnosis dificult. The nocturnal pains, in conjunction with the other symptoms present, induced a belief in the existence of the pulpy thickening of the synorial membrane, as spoken of by Bir B. Brodie; but the tolerably free motion, and the great hardiness, were opposed to this. The principal treatinent in the first inetance, was 
directed to the improvement of the general health; this was, in some degree, effected; her strength, however, failed; morning sweats and increased pain in the ham rendered it necessary to amputate the limb on the 17 th of September.

\section{Examination of the Joint.}

The cellular membrane covering the joint was very much thickened, and indurated, in some parts presenting a glandular appearance. The swelling commenced three inches above the condyles, and extended to their extremities; it was globular, and on cutting through the shaft of the femur longitudinally, it was found to be osteo-sarcoma. Several parts were softened, and contained small bony particles; in the ham, or rather between the condyles, where she complained of the pain, was a cavity containing about th ounce and half of coagulated blood; the skin covering this was discoloured; at the bottom of this cavity was the same description of bony particles as alluded to above ; the substance of the swelling was so hard, as to render it difficult to cut. The interior of the joint was healthy, with the exception of the internal ligaments, which were dark coloured and much thickened.

Since the operation the general health has much improved, and the stump is nearly healed.

\section{LIVERPOOL MEDICAL INSTITUTION.}

The first ordinary meeting of the members of the Liverpool Medical Institution was held on 'Thursday, the 1st of October, for the purpose of electing four members as vicepresidents, whose duties are to preside, in rotation, at the ordinary meetings of the institution. At these meetings papers are read, and medical intelligence received. There are upwards of 150 members of the institution, to which is attached a large and extensive library. Each member subscribes $2 l$. 2s. annually, and at least one-third of the amount must be expended in books; the remaining twothirds are appropriated to the incidental expenses of the building, which is very handsome, having a front of white stone; it was erected by public contributions, but principally by the munificence of the late Dr. Rutter. The gentlemen elected as vice-presidents were, Dr. Freckleton, Dr. James Vose, Dr. Duncan, and Mr. Banner. Dr. Sutherland was re-elected secretary.

\section{ST. MARYLEBONE INFIRMARY.}

CASES OF ABSCESSES OCCURRING IN THE BRAIN. BY R. BOY D, M.D. Resident Physician.

MARY S—, æ- at. 19, admitted in the last stage of phthisis, states that she has been subject to spitting of blood on making any exertion, for the last eleven years; has been getting gradually worse for the last three months; the spitting of blood during the last week amounted to about three pints. Her mother died under my care of phthisis about three years ago, at which period this girl had medicine prescribed for her, of which, or of her symptoms, no note was taken at the time. I am indebted to Dr. Harrison (whose patient she was in the Infirmary) for the following brief note of her symptoms on admission: pulse rather quick, jerking, compressible; skin hot; tongue whitish ; bowels open, though usually confined; catamenia ceased four months ago; severe head-ache during the last three days; nausea for the last week; vomited this morning some greenish coloured matter. On examining the chest, a dull sound was elicited by percussion, under the right clavicle,' with considerable flattening of that region, \&c. During the two following days the headache became pro-
gressively worse. Considerable excitement now existed, and she often shrieked out from the intensity of the pain. The vomiting still continued; pupils slightly dilated. Following day, more composed; pain less acute; seemed afraid to move her head from the pillow; spoke in an under tone. The next morning, little apparent change.
She could get out of bed without any assistance, when she had occasion to do so. About half an hour after this she died quite tranquilly.

\section{Autopsy 25 hours after Death.}

Head only allowed to be examined.-On removing the dura mater, a slight cohesion was found to exist betwixt the surfaces of the arachnoid, which presented a dull glazed appearance; it communicated to the touch a feeling of dryness, with unctuosity; the convolutions appeared flattened; veins considerably distended with blood. On the superior, posterior, and outer portion of the left hemisphere, was observed a depôt of purulent matter, as large as a shilling, extending into the substance of the brain. The anterior half of the body of the lateral ventricle was filled with pus, streaked with blood. On examination, a deposit about the same size as the first was found in the inferior and inner portion of the corpus striatum. A smaller deposit existed in the inferior, anterior, and outer portion of the left hemisphere ; and a fourth, still smaller, was found in the cortical substance of the upper and anterior portion of the right hemisphere. On the surface of the arachncid, at the junction of the pons Varolii with the medulla oblongata, there was a yellowish deposit, probably the result of inflammatory action. The interior of each of the cavities containing the purulent deposits, seemed to be lined with a distinct membrane; that lining the small cavity in the cortical substance was smoother than those of the others, which were all more or less irregular in their interior, especially that in the corpus striatum, on the inferior surface of which there were some enlarged vessels, and the pus in this situation was mixed with a little blood. The matter in all the cavities, on examination, presented the characters of healthy pus.

The symptoms of cerebral disturbance were only complained of eight days before death; and they did not arrive at their height till forty-eight hours before that event, when she presented all the symptoms of a person labouring under acute arachnitis. I recollect a male insane patient, under the care of the late Dr. Sims, exhibiting very similar symptoms, followed by death. He said at the time we should probably find a dryness of the arachnoid membrane. The post-mortem examination proved that his opinion was correct. The late Dr. Hooper, of this institution, has recorded a few cases of a similar nature. The works of Morgagni, Portal, Bailly, Abercrombie, \&c. furnish several examples. A case occurred in the practice of this infirmary, of abscess in the " pons Varolii," which was exhibited by Mr. Obré, at one of the early meetings of the Patholological Society last season. (Vide Lancet, and Medical Gazette, March 28th, 1840.) Such cases are, notwithstanding, very rare ; only two or three examples of it having occurred, out of many hundreds of examinations of cerebral complications made at this infirmary during the last six years. Cerebral symptoms at the termination of phthisis are by no means uncommon. From an analysis of fifteen cases of that disease, which were examined here during the autumn of 1839 , it appears, two cases were attended with delirium; in one of these there was softening of the septum lucidum, and about $3 \mathrm{oz}$. of fluid in the lateral ventricles. The other case also contained a somewhat smaller quantity of fluid in the lateral ventricles.

Dr. Clendinning, in one of his Clinical Lectures here last year, related the case of a young man, who was admitted into the infirmary in a state of delirium, and complaining by various intelligible gestures and cries, of intense pain of the head, without coma, or affection of the pupils, and making no other complaint, nor seeming to suffer at all otherwise. He was wasted, and had a slight cough, without expectoration. There was dulness on percussion, and depression of both subclavian regions. The restlessness of the patient prevented satisfactory auscultation; but the case needed no further light than that elicited by the fingers for decisive diagnosis. After ten days or a fortnight, he sank. The membranes of the brain, too, were congested, and pure serum, amounting to above $2 \mathrm{oz}$, existed in the ventricles. 